\title{
[A Current Bibliography of Canadian Church History]
}

Volume 42, 1975

URI : https://id.erudit.org/iderudit/1007238ar

DOI : https://doi.org/10.7202/1007238ar

Aller au sommaire du numéro

Éditeur(s)

Les Éditions Historia Ecclesiæ Catholicæ Canadensis Inc.

ISSN

0318-6172 (imprimé)

1927-7067 (numérique)

Découvrir la revue

Citer ce document

(1975). [A Current Bibliography of Canadian Church History]. Sessions d'étude -

Société canadienne d'histoire de l'Église catholique, 42, 111-141.

https://doi.org/10.7202/1007238ar

Tous droits réservés @ Les Éditions Historia Ecclesiæ Catholicæ Canadensis Inc., 1976
Ce document est protégé par la loi sur le droit d'auteur. L'utilisation des services d'Érudit (y compris la reproduction) est assujettie à sa politique d'utilisation que vous pouvez consulter en ligne.

https://apropos.erudit.org/fr/usagers/politique-dutilisation/ 


\section{GUIDES : bibliographies, descriptions of sources}

Artken, Barbara B. Local Histories of Ontario Municipalities Published in the Years 1957-1972. Kingston: Kingston Public Library Board, c1972. Pp. 50.

Andreassen, John Christian Ludwig. A Preliminary Guide to the Records of the Montreal General Hospital deposited in the McGill University Archives, accession no. 1501, 1818-1972. Montreal : University Archives, McGill University, 1972. Pp. 18.

Anschutz, Martha. A Preliminary List of Writings on Canadian Archives and Records Management. Montreal : University Archives, McGill University, 1970. Pp. 27.

"The Archival Organization of the United Church of Canada", The Bulletin, 22 (1973) 5-15.

BÉRUBÉ, Léo. "Les archives du diocèse de Rimouski", L'Église canadienne, 6 (1973) 124.

— "Le dépôt d'archives du diocèse de Rimouski", Archives, 73,2 (1973) 35-37.

Bissonnetre, André et André Frenière. "Alfred Charpentier : Biographie : Fonds Alfred Charpentier", Archives, 73,1 (1973) 4-12.

A Catalogue of Important Canadian Paintings, Drawings, Watercolours, Books and Prints of the 19th and 20th Centuries. Toronto: Sotheby and Co., 1972. Pp. 97.

CôTÉ, André. "Inventaire sommaire des sources manuscrites, conservées au Saguenay-Lac Saint-Jean”, Sessions d'étude, 40 (1973) 9-24.

DefoE, Deborah, comp. "Kingston: A Selected Bibliography", Historic Kingston, 21 (1973) 78-101.

DesJaRdins, Jeanne. "Scours de Notre-Dame du Saint-Rosaire, Rimouski : Archives : Cadre de classement", Archives, 73,1 (1973) 57-61.

Gagnon, Claire et François Rousseau. "Deux inventaires des archives de l'Hôtel-Dieu de Québec", Archives, 73,1 (1973) 62-82.

The Genealogical Society of the Church of Jesus Christ of Latter-Day Saints. Major Genealogical Record Sources for Canada. Research Paper Series B, no. 2. Revised edn. Salt Lake City: The Genealogical Society, 1972.

GresBrecht, Herbert, comp. The Mennonite Brethren : A Bibliographic Guide to Information. Fresno, California : Board of Christian Literature of Mennonite Brethren Churches, 1971. Pp. 17.

HanRahan, James. "A Current Bibliography of Canadian Church History", Study Sessions, 40 (1973) 69-93.

HoRvaTH, Maria, comp. and ed. A Doukhobor Bibliography, based on material collected in the University of British Columbia Library : Books and Periodical Articles. Enl. rev. edn. of Part I, Reference publication no. 22 (1968) and Supplement (1970). Vancouver: University of British Columbia Library, 1972. Pp. ca.150. 
KUEHL, Warren F. Dissertations in History : an index to dissertations completed in History Departments of United States and Canadian Universities 1961-June 1970. Lexington: University of Kentucky Press, c1972. Pp. $\mathbf{x}, 237$.

LAMONDE, Yvan. "Bibliographie des bibliographies des historiens canadiensfrançais du Québec", Recherches sociographiques, 12 (1971) 237-248.

Laurent, Monique. Le Catalogue de la bibliothèque du Séminaire de Québec, 1782. Thèse de D.E.S., Université Laval, 1973. Pp. xiv,101.

Leblanc, André E., et James D. Thwaites. Le Monde ouvrier au Québec: bibliographie rétrospective. Montréal: Les Presses de l'Université du Québec, 1972. Pp. xvi,283.

LinTEAU, Paul-André, et Jean Thivièrge. Montréal au 19 e siècle : bibliographie. Montréal : Université du Québec à Montréal, (1973). Polycopié.

NORTH, Robert, comp. "Bibliography of Works in Theology and History", History and Theory, 12 (1973) 55-140.

OWen, Myra, comp. Annual Reports, By-Laws and other Printed Documents of the Montreal General Hospital, 1823-1971, Accession no. 1501/9 and 1501/75/ University Archives, McGill University. Montreal : University Archives, McGill University, 1972. Pp. vii, 62.

Price, Brian J. "The Archives of the Archdiocese of Kingston", Study Sessions, 40 (1973) 21-25.

Prud'homme, François. "Clercs de Saint-Viateur, Province de Montréal : Cadre de classement", Archives, 73,1 (1973) 51-56.

PruItT, Sylvia, and Astrid Witschi-Bernz, comps. "Bibliography of Works in the Philosophy of History, 1969-1972", History and Theory, Beiheft 13 (1974) 1-106.

RYDER, Dorothy E. Canadian Reference Sources: A selective guide. Ottawa : Canadian Library Association, 1973. Pp. x,185.

Savard, Pierre. "Une bibliothèque idéale de défense religieuse au Canada français en 1887", Mélanges André Latreille, Lyon : Ed. Audin, 1972, 199-206.

Scollard, Robert Joseph, comp. A Bibliography of the Writings of Charles Collins, C.S.B. With an introduction by J. Francis Mallon, C.S.B. Toronto: The Basilian Press, 1974. Pp. 24. (Basilian Historical Bulletin, No. 9).

- "Roman Catholic History and Archives in the Province of Ontario", Families, 13,2 (Spring, 1974) 34-35.

Wilson, Ian E. "A Brief Guide to Archival Sources Relating to Kingston", Historic Kingston, 21 (1973) 76-77.

Winkler, Linda Mary. Principal William Peterson letterbooks, 1897-1919. Record Group 2P. Montreal: University Archives McGill University, 1973. Pp 22.

WOYCENKo, Ol'ha. Slov'yans'ki archivy $v$ Kanadi: Kolekciyi U.V.A.N. y Vinnipezi/Slavic Archives in Canada: UVAN Collections in Winnipeg. Winnipeg : UVAN, 1971. 


\section{SOURCES}

BÉLANGER, Léonidas, comp. "Les Mariages de la région", Saguenayensia, 14 (1972) 41-44, 69-72, 97-100, 173-176.

Bergeron, Roger, et Jean Bergeron, comps. Répertoire des mariages de la paroisse de La Visitation du Sault-au-Récollet, 1736-1970. Montréal : Roger et Jean Bergeron, 1973. Pp. 223.

- Répertoire des mariages des paroisses Sainte-Dorothée (1869-1970) et Saint-Elzéar-de-Laval (1900-1970). Montréal : Roger et Jean Bergeron, 1973. Pp. 112.

BoUlianNe, Henriette Laberge, comp. Répertoire des mariages de la paroisse de Sainte-Martine, co. Châteauguay, 1823-1972. Montréal: Roger et Jean Bergeron, 1973. Pp. 141.

CRÉPEAU, Pascal, et al., comps. Répertoire des mariages de la paroisse de St-Théodore de Chertsey, comté de Montcalm-Québec (1858-1972). Chertsey, 1973. Pp. 108.

Dessaules, Louis-Antoine. "Contre les détracteurs de l'Institut canadien", Études Françaises, 9,3 (août, 1973) 205-236.

LAflèche, Guy. Relation de 1634 de Paul Lejeune: Le missionnaire, l'apostat, le sorcier. Montréal : Les Presses de l'Université de Montréal, 1973. Pp. xli,261.

LeClerc, Paul-André, éd. "Mémoire sur la paroisse, le collège, le village, l'École d'Agriculture Sainte-Anne", Québec-Histoire, 1,1 (février-mars, 1971) 51-58.

LÉGARÉ, Jules, comp. Répertoire des mariages de Ste-Ursule (Cté. Maskinongé) 1842 à 1971. (Longueuil:) Jules Légaré, (1973). Pp. v, 225.

Legault, Maurice. Répertoire des mariages de l'île Perrot, comprenant SteJeanne-deJChantal (1786-1970), Ste-Rose-de-Lima, Brussy (1948-1970), Notre-Dame de Lorette, Pincourt (1948-1970), Notre-Dame de la Protection (1954-1970). Montréal : R. \& J. Bergeron, 1973. Pp. iv, 124.

Le journal des Jésuites. Publié d'après le manuscrit original conservé aux archives du Séminaire de Québec par MM. les abbés Laverdière et Casgrain. $3^{\mathrm{e}}$ éd., exactement conforme à la première (1871). Montréal : Éditions François-Xavier, 1973. Pp. x, 403, illus.

Pontbriand, Benoît. Mariages de Granby, comté de Shefford. 2 vols. Québec : B. Pontbriand, 1972.

- comp. Mariages du comté de Napierville: Napierville (1823-1970), StRémi (1831-1969), St-Édouard (1833-1969), Sherrington (1853-1966), St-Michel (1854-1969). Québec : B. Pontbriand, 1973. Pp. 451.

- comp. Mariages du comté de Shefford (1846-1968): Milton (1846), Stukely (1846), Roxton Falls (1850), Valcourt (1854), St-Valérien (1854), La Rochelle (1857), Bromont (1859), St-Joachim (1860), Waterloo (1865), Roxton Pond (1873), Maricourt (1893), Racine (1906), Béthanie (1916), Lawrenceville (1922). Québec : B. Pontbriand, 1973. Pp. 566. 
- et René Jetré, comps. Mariages de St-Hughes, 1827-1968, Théodore, 1842-1968, Ste-Hélène, 1854-1968, Upton, St-Ephrem, 1856-1968, Acton Vale, 1859-1968, Ste-Christine, 1886-1968, St-Nazaire, 1890-1968, comté de Bagot. Québec : B. Pontbriand, 1972. Pp. 375.

- et Raymond Gingras, comps. Mariages de St-Sylvestre, 1829, St-Gilles, 1830, Ste-Agathe, 1857, St-Patrice, 1860, St-Narcisse, 1873, comté de Lotbinière. Québec : B. Pontbriand, 1972. Pp. 242.

Proulx, Armand, comp. Répertoire des mariages de Notre-Dame-du-Rosaire, 1889-1971, de Sainte-Euphémie, 1907-1971, comté de Montmagny. La Pocatière, (1973). Pp. 59, 45.

- Répertoire des mariages de Saint-Adalbert, 1890-1971. Répertoire des mariages de Saint-Marcel, 1894-1971. La Pocatière : A. Proulx, 1973. Pp: 40, 44.

- Répertoire des mariages de Saint-Clément de Tourville, 1919-1971, comté de l'Islet. La Pocatière, (1973). Pp. vi, 52.

- Répertoire des mariages de Saint-Cyrille de l'Islet, 1865-1972. La Pocatière : A. Proulx, 1973. Pp. 108.

- Répertoire des mariages de Saint-Eugène de l'Islet: 1868-1972, $2^{e}$ édn.; de l'Isletville (St-Omer) 1962-1972. La Pocatière, (1973). Pp. 110, 12.

- Répertoire des mariages de Saint-Jean-Port-Joli, 1767 (i.e. 1779)-1973. La Pocatière, (1973). Pp. iii, 410.

- Répertoire des mariages de Saint-Just-de-Bretenière 1916-1971, de SainteLucie-de-Beauregard 1916-1971 comté de Montmagny, du Lac Frontière 1921-1971, de Saint-Fabien de Panet 1904-1971. La Pocatière, (1973). Pp. 40, 30, 19, 67.

- Répertoire des mariages de Saint-Pamphile, 1880-1972, de Saint-Omer, 1938-1972, comté de l'Islet. La Pocatière, (1973). Pp. 154, 17.

- Répertoire des mariages de Saint-Paul de Montminy (Montmagny) 18681972. La Pocatière, (1973). Pp. 116.

- Répertoire des mariages de Saint-Roch-des-Aulnets, 1734-1972. La Pocatière : A. Proulx, 1973. Pp. 36, vii, 353.

- Répertoire des mariages de Sainte-Apolline, 1902-1971, comté de Montmagny. La Pocatière, (1973). Pp. 55.

- Répertoire des mariages de Sainte-Perpétue, 1869-1972, de Sainte-Félicité, 1945-1971, comté de l'Islet. La Pocatière, (1973). Pp. 131, iv, 16.

Provencher, G. E. Mariages de l'Outaouais (Vol. V): L'Orignal, 1836-1882, Curran, 1839-1961, Clarence Creek, 1855-1945, Orléans, 1859-1867, Rockland, 1887-1906, Ontario. Québec : B. Pontbriand, 1972.

- et M. Langlors, comps. Mariages de l'Outaouais (Vol. IV): Ottawa, N.-D., 1829-1880, Ottawa, Ste-Anne, 1873-1900, Ottawa, Notre-Dame du Bon Conseil, 1889-1907. Québec: B. Pontbriand, 1972.

REIBIN, S. "Report of General Meeting of the Doukhobor Community Held at Verigin, January 25th, 1910", Saskatchewan History, 24 (1971) 73-75. (Reprinted from Free Press, Winnipeg, March 1, 1910.)

Relations des Jésuites, 1611-1672. 6 vols. Montréal : Éditions du jour, 1972. (Édition originale: Québec, 1858.) 
ROBERge, Claude, comp. Répertoire des mariages de Saint-Charles de Limoilou, Québec, 1896-1971. (Québec:) Société canadienne de généalogie (Québec), 1973. Pp. 226.

- Répertoire des mariages du Cap-de-la-Madeleine, Ste-Marie-Madeleine, 1673-1971. (Québec:) Société canadienne de généalogie (Québec), 1973. Pp. 217.

\section{GENERAL WORKS}

See above : 1. Kuehl; Lamonde.

Beaulieu, André, Jean Hamelin, Benoît Bernier. Guide d'histoire du Canada. Québec: Les Presses de l'Université Laval, 1972. Pp. xvi, 544.

Brunet, Michel. Précis d'histoire du Canada de la Nouvelle-France à nos jours. [Montréal], Librairie de l'Université de Montréal, 1972. Pp. 76.

Burnet, Jean R. Ethnic Groups in Upper Canada. Toronto : Ontario Historical Society, 1972.

DORge, Lionel. Introduction à l'étude des Franco-manitobains. Saint-Boniface : La Société historique de Saint-Boniface, 1973. Pp. v, 298.

Dumas, Silvio. Les Filles du Roi en Nouvelle-France. Québec : La Société historique de Québec, 1972.

"Le fait français dans le Nord de l'Ontario", Revue de l'Université Laurentienne, 3,4 (juin, 1971) 1-106.

Howard, Richard, Jacques Lacoursière et Claude Bouchard. A New History of Canada. 15 vols. Montréal : Éditions Format, 1973. Pp. 1438, illus.

JACKson, Michael. "Une minorité ignorée : les Franco-Canadiens de la Saskatchewan", Journal of Canadian Studies/Revue d'études canadiennes, 7:3 (August 1972 août) 1-20.

Lacoursière, Jacques et Claude Bouchard. Notre Histoire : Québec-Canada. 15 volumes. Montréal : Éditions Format, 1972. Pp. 1438, illus.

MORRISON, William Robert. The Mounted Police on Canada's Northern Frontier, 1895-1940. Ph.D. Thesis, University of Western Ontario, 1973. Diss Abs, 34 (1973-74) 1832-A.

NORRIS, John. Strangers Entertained : A History of the Ethnic Groups of British Columbia. Vancouver: British Columbia Centennial '71 Committee, 1971. Pp. 254.

Ouellet, Fernand. Éléments d'histoire sociale du Bas-Canada. Montréal : Hurtubise HMH, 1972. Pp. 379

Palmer, Howard. The Land of Second Chance: A History of Ethnic Groups in Southern Alberta. Lethbridge : Lethbridge Herald, 1972. Pp. 287.

Paquet, Gilles, et Jean-Pierre Wallot. "Groupes sociaux et pouvoir : le cas canadien au tournant du XIXe siècle", RHAF, 27 (1973-1974) 509-.

Rioux, Marcel, et Yves MaRtin, éds. La société canadienne-française. Montréal : HMH, 1971. Pp. 444. 
Rumilly, Robert. Histoire de la province de Québec. 4: Les "Castors". Montréal : Fides, 1973.

- Histoire de Montréal. 3 vols. Montréal : Fides, 1970-1972.

Russell, Loris S. Everyday Life in Colonial Canada. Toronto : Copp Clark Pub. Co., 1973. Pp. 206.

SÉGUIN, Robert-Lionel, et al. Ethnologie québécoise. Tome 1. Montréal : Hurtubise HMH, 1973.

Trofimenkoff, S. M. The Twenties in Western Canada. Ottawa: Musée national de l'homme, 1972.

Trudel, Marcel. The Beginnings of New France, 1524-1663. Translated by Patricia Claxton. Toronto: McClelland and Stewart, c1973. Pp. xii, 323, illus.

— "Les obligations du censitaire à l'époque des Cent-Associés", $R H A F$, 27 (1973-74) 3-41.

VoyCEnKo, Ol'ha. Do istoryi ukrainciv u Brytans'ky Kol'umbiyi/Ukrainians in British Columbia, a Page from their History. Winnipeg: Ukrainian Free Academy of Sciences, 1972. Pp. 46, illus.

WISE, S. F. "Liberal Consensus or Ideological Battleground : Some Reflections on the Hartz Thesis", HPCH, (1974) 1-14.

\section{CHURCH HISTORY : philosophy, principles}

See above : 1 . North; Pruitt.

DOHERTY, Robert W. "Sociology, Religion, and Historians", Historical Methods Newsletter, 6,4 (September, 1973) 161-169.

Fitzsimons, M. A. "The Role of Providence in History", Review of Politics, 35 (1973) 386-397.

Grant, George. Time as History. Toronto : Canadian Broadcasting Corporation, 1971. Pp. x, 52.

KIM, J. J. "Belief or Anamnesis : Is a Rapprochement between History of Religion and Theology Possible?", Journal of Religion, 52 (1972) 150169.

Landes, David S., Charles Tilly, Howard F. Cline, et al. History as Social Science. Englewood Cliffs, N.J. : Prentice-Hall, 1971. Pp. viii, 152.

Le Roy Ladurie, Emmanuel. Le territoire de l'historien. Paris: Gallimard, 1973. Pp. 542.

Patrides, C. A. The Grand Design of God: The Literary Form of the Christian View of History. London : Routledge and K. Paul; Toronto : University of Toronto Press, 1972. Pp. xvii, 157, illus.

Richardson, W. J. “A Christian View of Progress", Thought, 46 (1971) 562 576.

ROBERTSON, Roland. The Sociological Interpretation of Religion. New York : Schocken Books Inc., 1970. Pp. 256. 
SÉGUY, Jean. "Max Weber et la sociologie historique des religions", Archives de Sociologie des Religions, 17,33 (1972) 71-104.

ShorTer, Edward, The Historian and the Computer. Englewood Cliffs, N.J. : Prentice-Hall, 1971. Pp. x, 149.

SrRINZak, Ehud. "Weber's Thesis as an Historical Explanation", History and Theory, 11 (1972) 294-320.

VeYne, Paul. Comment on écrit l'histoire: Essai d'épistémologie. Paris : Éditions du Seuil, 1971. Pp. 350.

Watelet, Hubert. "Connaissance et sociologie de la connaissance chez les historiens : À propos de l'article de Serge Gagnon", RHAF, 27 (19731974) 571-578.

\section{THE COMMUNIONS}

\section{A. General}

ProulX, Jean-Pierre. "Les églises québécoises, de Durham à nos jours", Le Devoir (9 déc., 1972) 15, 19.

PuXley, H. L. "Ecumenism in Canada", Église et Théologie, 2 (1971) 397409.

\section{B. Particular Communions}

\section{Anglican}

ERvin, Spencer. The Development of the Synodical System of the Anglican Church of Canada. Ambler, Pa. : Trinity Press, c1969. Pp. xii, 25.

\section{Baptist}

Carder, W. Gordon. "Controversy in the Baptist Convention of Ontario and Quebec, 1908-1928”, Foundations, 16 (1973) 355-376.

KINDRAT, Petro. Ukrains'kyj baptysts'kyi rukh u Kanadi/Ukrainian Baptist Movement in Canada. Winnipeg: Doroha Pravdi, 1972. Pp. 172.

LANGLeY, Thelma, ed. Baptist Family in Global Village. (Toronto) : Canadian Baptist Overseas Mission Board for the Committee on Missionary Education, the Baptist Federation of Canada, 1972. Pp. 173, illus.

Zeman, Jarold K. "Baptists in Canada and Co-Operative Christianity", Foundations, 15 (July-Sept., 1972) 221-240.

\section{Children of Peace (Davidite)}

Classey, Tim. "The Old Pipe Organ", The York Pioneer, (1974) 67-69.

McFall, Jean. "The Last Days of the Children of Peace", The York Pioneer, (1973) 22-33.

McIntYre, W. John. "David Willson and Government, Politics and Society in Upper Canada", The York Pioneer, (1974) 2-15.

\section{Doukhobor}

See above : 1. Horvath.

2. Reibin. 
Stoochnoff, John Philip. Toil and Peaceful Life: Doukhobors as They Are. 2nd rev. edn. Vancouver : Liberty Press, 1971. Pp. 117, illus.

VARNALS, D. "The Doukhobors in the Kootenays: Signs of Stress", B.C. Perspectives, 3 (March, 1973) 15-20.

\section{Jewish}

Arnold, J. A. "The Jews of Canada - The Prairies", View Points - Canadian Jewish Quarterly, 7,3-4 (1973) 63-83.

GOEB, Joan. "The Jews of Canada - The Maritimes", View Points - Canadian Jewish Quarterly, 7,3-4 (1973) 9-23.

GUTwIRTH, Jacques. "Hassidim et judaïcité à Montréal", Recherches sociographiques, 14 (1973) 291-325.

HAYes, Saul. “A View on Canadian Jewish History", View Points - Canadian Jewish Quarterly, 7,3-4 (1973) 6-8.

Helfield, Tilya Gallay. "The Jews of Canada-Québec", View Points Canadian Jewish Quarterly, 7,3-4 (1973) 24-51.

KaYfetz, Ben. "The Jews of Canada - Ontario", View Points - Canadian Jewish Quarterly, 7,3-4 (1973) 52-62.

RrvkIN, Ann. "The Jews of Canada - British Columbia", View Points Canadian Jewish Quarterly, 7,3-4 (1973) 84-95.

Teboul, Victor, et Salomon Schinasi. "La Juive québécoise", Actualité, 11,11 (novembre 1971) 60-66.

\section{Mennonite}

See above : 1 . Giesbrecht.

DRIEDGER, Leo. "Native Rebellion and Mennonite Invasion : An Examination of Two Canadian River Valleys", The Mennonite Quarterly Review, 46 (1972) 290-300.

- and Jacob Peters. "Ethnic Identity : A Comparison of Mennonite and other German Students", The Mennonite Quarterly Review, 47 (1973) 225-244.

Gingerich, Orland. The Amish of Canada. Waterloo, Ont.: Conrad Press, c1972. Pp. 244, illus.

PoETTCKer, Henry. Call to Faithfulness: Essays in Canadian Mennonite Studies. Winnipeg: Canadian Mennonite Bible College, c1972. Pp. xiv, 222.

RoTH, Lorraine. "150 Years" : The Story of the Amish Mennonites of Ontario. New Hamburg, Ont. : Mennonite Historical Committee, 1972.

\section{Methodist}

Brooks, William Howard. Methodism in the Canadian West in the Nineteenth Century. Ph.D. Thesis, University of Manitoba, 1972. Pp. xiii, 403. Canadian Theses on Microfilm, no. 12651.

EMERY, G. N. "The Methodist Church and the 'European Foreigners' of Winnipeg : the All People's Mission, 1889-1914", Historical and Scientific Society of Manitoba Transactions, 28 (1971-1972) 85-100. 
LindSTROM, Erling Godfred. The Methodist Church, Canada, 1884-1912. M.A. Thesis, Simon Fraser University, 1972. Pp. vii, 154. Canadian Theses on Microfilm, no. 12137.

\section{Moravian}

BRUEMMER, Fred. "Northern Labrador", Canadian Geographical Journal, 82 (Jan.-June, 1971) 158-167, illus.

Hamilton, J. Taylor. A History of the Church Known as the Moravian Church. Bethlehem, Pa.: Times Publishing Company, 1900; New York: AMS Press, 1971. Pp. xi, 632.

HILLER, J. K. The Foundation and the Early Years of the Moravian Mission in Labrador, 1752-1805. M.A. Thesis, Memorial University of Newfoundland, 1968. Pp. xii, 246. Canadian Theses on Microfilm, no. 14313.

\section{Quaker}

Dorland, Arthur Garratt. Former Days and Quaker Ways: A Canadian Retrospect. 2nd edn. Belleville, Ont.: Mika Studio, 1972. Pp. ix, 198, illus. (Canadiana Reprint Series, no. 41.)

Fell, Brian John. A Question of Conscience: British and Canadian Quakers and their Socialist and Parliamentary Allies Face the Great War. M.A. Thesis, University of Manitoba, 1969. Pp. 232. Canadian Theses on Microfilm, no. 11467.

\section{United Church of Canada}

See above : 1. Archival.

Hoввs, R. Gerald. "The Nature and Exercise of Authority in the United Church of Canada", The Bulletin, 22 (1973) 21-35.

Plato, W. R. "The United Church of Canada and Eucharistic Worship", The Bulletin, 22 (1973) 16-20.

\section{REGIONAL HISTORY}

\section{A. Diocese, Congregation, Synod}

\section{Alberta}

See above : 3. Palmer.

\section{British Columbia}

See above : 3. Norris; Voycenko.

5B. Jewish-Rivkin.

\section{Calgary, Alta.}

BYRNE, M. B. From the Buffalo to the Cross: A History of the Roman Catholic Diocese of Calgary. Calgary : Calgary Archives and Historical Publishers, 1973. Pp. 555.

\section{Chicoutimi, Qué.}

Bélanger, René. "Le Séminaire de Chicoutimi et la Côte Nord, 1883-1903", Saguenayensia, 14 (1972) 136-137. 
Drolet, Jean-Claude. "Le chapitre de Chicoutimi", Saguenayensia, 14 (1972) 2-5.

— "Un collège-séminaire à Chicoutimi en 1873", Saguenayensia, 14 (1972) 118-134.

— "Le Séminaire de Chicoutimi, 1877-1883", Saguenayensia, 14 (1972) 158-168.

— "Souvenirs d'un serviteur : M. Wilfrid Boivin", Saguenayensia, 14 (1972) 146-147.

Une gerbe de souvenirs 1873-1973; ou, Le Séminaire raconté par ses Anciens. [Chicoutimi : Éditions Science moderne, 1973.] Pp. 211, illus.

LALIBERTÉ, Jean-Paul. "L'évolution du Séminaire de Chicoutimi", Sessions d'étude, 40 (1973) 131-134.

PARÉ, Marius. "Le Centenaire du Séminaire de Chicoutimi", Saguenayensia, 14 (1972) 116-117.

- "Le rôle des évêques de Chicoutimi dans l'œuvre du Séminaire", Sessions d'étude, 40 (1973) 113-124.

Pedneault, Roch. "L'évolution du Séminaire de Chicoutimi", Sessions d'étude, 40 (1973) 135-138.

Simard, Jean-Paul, et Bérard Riverin. "Origine géographique et sociale des étudiants du petit séminaire de Chicoutimi et leur orientation socioprofessionnelle: 1873-1930", Sessions d'étıde, 40 (1973) 33-53.

SimarD, O.-D. "Séminaire de Chicoutimi, 1873-1973 : Coup d'œil sur le siècle écoulé", Sessions d'étude, 40 (1973) 125-129.

Tremblay, Victor. "La fanfare du Séminaire, 1879-1912", Saguenayensia, 14 (1972) 185-189.

- "Une galerie d'employés", Saguenayensia, 14 (1972) 142-145.

- "Mes chantiers", Saguenayensia, 14 (1972) 149-153.

- "Le Séminaire de Chicoutimi : Bref aperçu de son histoire". Saguenayensia, 14 (1972) 114-115.

\section{Kingston, Ont.}

See above : 1. Defoe; Price; Wilson.

FLYNN, L.J. At School in Kingston 1850-1973. Frontenac, Lennox and Addington County Roman Catholic Separate School Board, 1973.

\section{Manitoba}

See above : 3. Dorge.

5B. Mennonite-Driedger (2).

\section{Maritime Provinces}

See above : 5B. Jewish-Goeb.

Montréal, Qué.

See above : 1. Linteau.

2. Rumilly.

5B. Jewish-Gutwirth. 
Jolın, Jean-Pierre. Mgr Édouard-Charles Fabre et le diocèse de Montréal, la question d'un coadjuteur à l'évêque de Montréal (1872-1873) et la question de l'érection de Montréal en archevêché (1879-1887): aperçu des relations interépiscopales. Thèse de M.A., Université McGill, 1972. $P p$. viii, $x, 86,34$. Thèses canadiennes sur microfilm, no. 11858.

Newfoundland

Sparkes, Paul. "Churches in a Land of Fishing Flakes", Atlantic Advocate, 63 (Sept., 1972) 23-25.

\section{Ontario}

See above : 1. Aitken; Scollard.

2. Fait.

5B. Jewish-Kayfetz. Mennonite-Gingerich; Roth.

\section{Prince Edward Island}

Macandrew, Barbara. "Oldest P.E.I. Anglican Church Gets a Face-Lifting", Atlantic Advocate, 63 (Nov., 1972) 13.

\section{Québec}

See above: 5A. Proulx.

5B. Jewish-Helfield; Teboul.

Bonenfant, Jean-Charles. "Une querelle paroissiale à l'île d'Orléans", Les cahiers des Dix, 38 (1973) 33-58.

Charbonneau, H. et Y. Lavoie. "Cartographie du premier découpage territorial des paroisses du Québec, 1721-1722", La Revue de géographie de Montréal, 27, 1 (1973) 81-87.

Le diocèse de Québec 1674-1974. Exposition: 31 janvier-3 mars 1974. Québec : Ministère des affaires culturelles, 1974. Pp. 59, illus.

LabrèQue, Marie-Paule. "L'Église dans les Cantons de l'Est (1800-1860)", Sessions d'études, 41 (1974) 87-103.

LAFRAmboise, Yves et Robart Derome. "Les manuscrits d'Isidore Desnoyers : Une histoire des paroisses de la vallée du Richelieu", Documents: Arts anciens du Québec, 1 (février, 1971) 23-26.

Savard, Pierre. "Sur les noms de paroisses au Québec, des origines à 1925", Sessions d'étude, 41 (1974) 105-.

\section{Rimouski, Qué.}

See above: 1. Bérubé (2).

Drapeau, Jean, et Rodrigue Bélanger. Une Église d'hier a demain : exploration et essais. Montréal: Presses de l'Université du Québec, 1973. Pp. xi, 195.

Saint Paul, Alta.

Drouin, E. O. Échos argentins au diocèse de Saint-Paul-en-Alberta. Edmonton : Éditions de l'Ermitage, 1973. 


\section{Sherbrooke, Qué.}

Lavallée, Jean-Guy. "L'Église de Sherbrooke et les Trappistes (1880-1948)", Sessions d'étude, 41 (1974) 9-24.

Vorsine, Nive. "L'épiscopat québécois au moment de la formation du diocèse de Sherbrooke (1874)", Sessions d'étude, 41 (1974) 25-41.

\section{Western Canada}

See above: 3. Jackson; Trofimenkoff.

5B. Jewish-Arnold;

Methodist-Brooks.

\section{Winnipeg}

See above: 5B. Methodist-Emery.

\section{B. Parish History}

Bécancour, Qué.

HÉbert, Pierre-Maurice. "Saint Grégoire (ville de Bécancour, comté et diocèse de Nicolet) 2e partie : la paroisse", Société historique acadienne, 4, 10 (juill.-août-sept., 1973) 425-439.

\section{Calgary, Alta.}

CARTer, David J. Calgary's Anglican Cathedral. Calgary: Kyle Printing and Stationery Ltd., 1973. Pp. 64, illus.

\section{Cookshire, Qué.}

Saint-Camille de Lellis, 1868/1968. Cookshire, Qué., [1968]. Pp. 56.

\section{Courtland, Ont.}

O'NeIL, J. H. Diary of a Priest (Containing a History of Catholicism of Tillsonburg, Norwich, Langton, Port Burwell and Courtland.) 1970.

\section{Florenceville, N.B.}

United Church of Canada, Florenceville Pastoral Charge. The United Church of Canada, Florenceville, N.B. ce'ebrates the 100th anniversary of the erection of the church building, Sunday, November 2nd, 1969, 11:30 A.M. (Florenceville, N.B.: Florenceville Pastoral Charge of the United Church of Canada, 1971.) Pp. 32, illus.

\section{Fredericton, N.B.}

"180th Anniversary", Atlantic Advocate, 62 (Jan., 1972) 47. (Wilmot Church.)

\section{Grimsby, Ont.}

Bromley, Ada and Jean Powell. History of Trinity United Church, 18011971. Grimsby, Ont.: Trinity United Church, 1971. Pp. 58, illus.

\section{Halifax, N.S.}

SINClaIR, Donald Maclean. Fort Massey Church, Halifax, Nova Scotia, 1871-1971: a century of witness. (s.l. : s.n., 1971.) Pp. vi, 49. 
Hamilton, Ont.

Anderson, Frederick Keith and John B. McMnlan. A History of James St. Baptist Church, 1844-1969. Hamilton, Ont., 1969.

Harbour Grace, Nfld.

Davis, May. "The Story of Education at St. Paul's Parish, Harbour Grace", Newfoundland Quarterly, 69 (Dec., 1972) 4-5.

Kingston, Ont.

FLYNN, Louis J. "The History of Saint Mary's Cathedral of the Immaculate Conception, Kingston, Ontario, 1843-1973", Study Sessions, 40 (1973) $35-40$.

Landestreu, Sask.

THREINEN, Norman J. Immanuel Evangelical Lutheran Church, Landestreu, 1895-1970. 1970. Pp. 28, illus.

Langton, Ont.

See above : 6B. Courtland.

Leamington, Ont.

DRIEDgER, N. N. The Leamington United Mennonite Church: Establishment and Development, 1925-1972. [s.l. : s.n., 1973.] Pp. viii, 176, illus.

- Die Leamingtoner Vereinigte Mennonitengemeinde: Enstehung und Werdegang, 1925-1972. (Leamington, Ont.) : Leamingtoner Vereinigte Mennonitengemeinde, [1973]. Pp. xii, 197, illus.

Medicine Hat, Alta.

Downes, Margaret. History of St. John's Presbyterian Chuch, Medicine Hat, Alberta, 1883-1973. Medicine Hat, Alta. : Session and Board of Managers, St. John's Presbyterian Church, [1973]. Pp. vii, 134, illus.

St. John's Presbyterian Church, Medicine Hat, Alberta, June 3, 1883-June 3, 1973; 90th Anniversary Celebrations, June 2 and 3, 1973. [Medicine Hat: St. John's Presbyterian Church,] 1973. Pp. 11.

North Surrey, B.C.

25-littya Ukrains'koyi pravoslavnoyi hromady uspinnya Presv. Bohorodytsi $v$ Valey-Surey, B.K., 1946-1971/25th Anniversary of St. Mary's Ukrainian Greek Orthodox Church, North Surrey, B.C. 1946-1971. Published by the Church's Jubilee Committee, 1971. Pp. 92, illus.

Norwich, Ont.

See above : 6B. Courtland.

Notre-Dame-des-Monts, Qué.

GIRARD, Judith Turcotte. La paroisse aux trois églises. Chicoutimi, Qué.: Éditions Science moderne, 1973. Pp. 206, illus.

\section{Ottawa, Ont.}

Hubbard, R. H. Cathedral in the Capital: A Short History of Christ Church Cathedral, Ottawa. Ottawa: Cathedral Centenary Committee, 1972. Pp. 86, illus. 
Plourde, Antonin-M. S.-Jean-Baptiste d'Ottawa, 1872-1972. [Ottawa ?] : A. Plourde, [1972]. Pp. 45, illus.

Port Burwell, Ont.

See above: 6B. Courtland.

Sainte-Anne-de-Beaupré, Qué.

Tremblay, Gérard. 100 ans dans le bon vent. [Sainte-Anne-de-Beaupré :] Revue Sainte Anne de Beaupré, [1973]. Pp. 69, illus.

Sainte-Anne-de-la-Pocatière, Qué.

See above : 2 . Leclerc.

Saint Charles, N.B.

Cent ans de vie paroissiale à Saint-Charles Borromée, 1872-1972. Pp. 22, illus.

Saint-Vianney, Qué.

BéRUBÉ, Léo. Saint-Vianney de Matapédia. Saint-Vianney, Québec : Comité des fêtes du cinquantenaire, 1972. Pp. 259, illus.

Salaberry-de-Valleyfield, Qué.

ST-AUBIN, Joseph-Damien. Salaberry-de-Valleyfield, 1842 à 1972 : histoire religieuse, municipale, scolaire, commerciale et industrielle. Valleyfield: Impr. G. Brault, 1972. Pp. 219.

Sorel, Qué.

Poulin, Gonzalve. La paroisse Saint-Maxime de Sorel, 1946-1971. Sorel : G. Poulin, 1971. Pp. 73, illus.

Tillsonburg, Ont.

See above : 6B. Courtland.

Toronto, Ont.

Milne, Dorothy. "York Mills Baptist Church", The York Pioneer, (1973) 17-21.

\section{Yarmouth, N.S.}

Blauveldt, Robert Brooks. Holy Trinity Anglican Church, Yarmouth, N.S., with a Brief Outline of the Growth of Anglicanism in Yarmouth since 1785. Yarmouth: Sentinel Printing, 1972. Pp. 48, illus.

\section{INSTITUTIONS}

See above : 1. Andreassen; Desjardins; Gagnon; Laurent; Owen; Prud'homme.

2. Dessaules.

6A. Chicoutimi-Bélanger; Chicoutimi-Drolet (3); ChicoutimiGerbe; Chicoutimi-Laliberté; Chicoutimi-Paré (2); Chicoutimi-Pedneault; Chicoutimi-Simard (2); Chicoutimi-Tremblay (4); Sherbrooke-Lavallée.

AgNew, G. Harvey. Canadian Hospitals, 1920 to 1970: A Dramatic Half Century. Toronto: University of Toronto Press, 1973. Pp. 288. illus. 
AUDET, Louis Philippe. "Bilan de la contribution de la section des Lettres et des Sciences humaines aux études canadiennes, 1952-1972", TRSC/MSRC, 11 (1973) 131-146.

Begnal, Calista. "Sisters of the Congregation of Notre-Dame, Nineteenth Century Kingston", Study Sessions, 40 (1973) 27-33.

Carrière, Gaston. "Les quarante ans de la Société canadienne d'Histoire de l'Église catholique", RUO, 43 (1973) 485-493.

- "Les quarante ans de la Société canadienne d'Histoire de l'Église catholique (1933-1973)", Sessions d'étude, 40 (1973) 25-32.

CôtÉ, André. "Le Monastère de Mistassini : sa suppression ou sa formation en prieuré, 1900-1903", Sessions d'étude, 40 (1973) 92-111.

D'Allaire, Micheline. L'Hôpital-Général de Québec, 1692-1764. Montréal : Fides, 1971. Pp. 254.

DESJARDINS, Édouard. "L'Hôpital de Jeanne Mance : 1642-1673", L'Union médicale du Canada, 102: 5 (mai, 1973) 1136-1142.

- "L'Hôpital de la Miséricorde à Montréal", L'Union médicale du Canada, 102 : 2 (février, 1973) 400-405.

Dewolf, Mark and George Flie. 1789: All the King's Men: The Story of a Colonial University. Halifax : Alumni Association of the University of King's College, c 1972. Pp. 58, illus.

Drolet, Jean-Claude. "Jalons pour une histoire de la Congrégation des Sœurs de Notre-Dame du Bon-Conseil de Chicoutimi”, Saguenayensia, 13 (1971) 156-160.

- "L'Ordre des Chevaliers de Colomb : Origine historique", Saguenayensia, 14 (1972) 45-48.

- "L'Ordre des Filles d'Isabelle", Saguenayensia, 14 (1972) 182-183.

"Les Frères du Sacré-Cour sont au Canada depuis cent ans", L'Église canadienne, 5 (1972) 172-173.

Gibson, Rose Mary. St. Mary's of the Lake in Kingston. Kingston : Maxwell Printers, 1971.

Guitard, Michelle. "Pour une histoire de l'Institut canadien de Montréal", RHAF, 27 (1973-1974) 403-407.

"L'Hôtel-Dieu de Montréal d'hier à demain", Culture Vivante, 28 (juin, 1973) 17-20.

JAmes, F. Cyril. McGill Milestones. (Montreal : Information Office, McGill University, 1972.) Pp. 7.

JEANNE-DE-FrancE, sœur. Route de vie, cheminement d'une formule d'éducation: l'Institut familial bilingue, Saint-Jacques, Montcalm, Qué. Lachine, Qué. : Maison-Mère, (Cong. des Sœurs de Sainte-Anne), 1972. Pp. xv, 235, illus.

La Palm, Loretta. "The Hôtel-Dieu of Québec: The First Hospital North of the Rio Grande under its First Two Superiors", Study Sessions, 41 (1974) 53-64. 
LEEFE, John. "King's and Dalhousie: An Early Attempt at University Consolidation in Nova Scotia", The Nova Scotia Historical Quarterly, 2 (1972) 41-54.

LÉGARÉ, Romain. Les 75 ans de la Fraternité Saint-Antoine (Laïcat Franciscain de Montréal) 1896-1971. Pp. 57.

MARYAN, Sister. "The Work of the Congregation of the Good Shepherd in Toronto, 1875-1973", The York Pioneer, (1974) 38-48.

Neatby, Hilda. "Queen's College and the Scottish Fact", Queen's Quarterly, 80 (1973) 1-11.

O'Farrell, John K. A. "Canadian Catholic Historical Association's Fortieth Anniversary : A Retrospective View", Study Sessions, 40 (1973) 61-68.

Pariseau, Claude. La bibliothèque du Collège de Québec. [Montréal]: Graduate School of Library Science, McGill University, 1972. Pp. iii, 24, illus.

Penton, D. S. Non nobis solum: The History of Lower Canada College and its Predecessor, St. John's School. Montreal : Corporation of Lower Canada College, c1972. Pp. xii, 336, illus.

Plourde, J. Antonin. Dominicains au Canada : livre des documents. 1 : La fondation maskoutaine, 1830-1886. Montréal : Éditions du Lévrier, 1973.

SENCIK, O. Stefan. Nase nové Slovenske duchavné stredisko", Slovenski Jesuiti v Kanada, (1973) 3-13.

Trudel, Marcel. "Les débuts de l'Institut d'histoire à l'Université Laval", RHAF, 27 (1973-1974) 397-402.

\section{INDIVIDUAL BIOGRAPHY AND BIOGRAPHICAL MATERIAL}

\section{A. General}

See above : 6A. Sherbrooke-Voisine.

Allan, Iris. "The McDougalls, Pioneers of the Plains", The Beaver, outfit 304, 1 (Summer, 1973) 14-19.

COPWAY, George. The Traditional History and Characteristic Sketches of the Ojibway Nation. London, Edinburgh, Dublin, 1850 ; Toronto : Coles Pub. Co., c1972. Pp. xii, 298.

CORNEZ, Germaine. "Saint-Jerôme et les lions du Nord", Québec-Histoire, 1, 5-6 (mai-juillet, 1972) 83-85.

Houde, Roland. "Jacques et Raïssa Maritain au Québec", Relations, 384 (juillet-août, 1973) 214-217.

McDougall, John M. Opening the Great West. Introduction by J. E. Nix. Calgary : Calgary Glenbow Alberta Institute, 1970. Pp. 63, illus.

SCOLlaRD, Robert Joseph. A Calendar of the Deceased Bishops and Priests of the Archdiocese of Toronto, December 1841-May 1974. Toronto, 1974. Pp. 16. 


\section{B. Individuals}

\section{Beaudry, R.P. René}

Chiasson, Anselme. "Le révérend père René Beaudry", La Société Historique Acadienne, 36, 4 (juillet-sept., 1972) 248-249.

Bégin, Card. Louis-Nazaire

Drolet, Jean-Claude. "Mgr. L.-N. Bégin (1818-1892) deuxième évêque de Chicoutimi”, Saguenayensia, 13 (1971) 39-42.

\section{Belley, Sœur Marie-Louise}

Tremblay, Victor. "L'odyssée d'une Saguenéenne: Sœur Marie-Louise Belley", Saguenayensia, 14 (1972) 89-92.

\section{Boucher, Cyrille}

SylvaIN, Philippe. "Cyrille Boucher (1834-1865) disciple de Louis Veuillot", Les cahiers des Dix, 37 (1972) 295-317.

\section{Bouillon, Abbé Alexandre}

Roy, Jeanne. Un pauve de Yahweh: Alexandre Bouillon (1873-1943), premier curé de la paroisse de Lac-au-Saumon et fondateur des Servantes de Notre-Dame, Reine du clergé. Lac-au-Saumon: [Maison générale Notre-Dame, Reine du clergé], 1973. Pp. 200, illus.

\section{Bourassa, Napoléon}

LE MoINe, Roger. Napoléon Bourassa. (Collection "Classiques canadiens", 44), Montréal : Fides, 1972. Pp. 88.

Bruchési, Mgr. Paul-Napoléon

BRUCHÉsI, Jean. "Un inlassable épistolier : Paul-Napoléon Bruchési, (18551939)", TRSC/MSRC, 10 (1972) 115-126.

\section{Bullock, Dean William}

Thомas, C. E. "Dean William Bullock, D.D.", Collections of the Nova Scotia Historical Society, 37 (1970) 23-37.

\section{Casault, Abbé Louis-Jacques}

SYlvaIN, Philippe. "Louis-Jacques Casault, fondateur de l'Université Laval", Les cahiers des Dix, 38 (1973) 117-132.

\section{Charpentier, Alfred}

See above: 1. Bissonnette.

Collins, Rev. Charles, C.S.B.

See above: 1. Scollard.

\section{Compain, Abbé Pierre-Joseph}

Bélanger, René. "L'abbé Pierre-Joseph Compain, prêtre et médecin, 17401806", Saguenayensia, 13 (1971) 106-107.

\section{Courchesne, Mgr. Georges}

Bélanger, Noël. Mgr. Courchesne et la vie rurale", Revue d'histoire du Bas-St-Laurent, 1, 1 (oct., 1973) 14-17. 


\section{Drolet, Antonio}

Boissonnault, Charles-Marie. "Antonio Drolet, bibliothécaire et historien", TRSC/MSRC, 10 (1972) 127-134.

Fabre, Mgr. Édouard-Charles

JetrÉ, Pierre. Le journal Canada-Revue et Mgr. Édouard-Charles Fabre, 1890-1895. Thèse de M.A. Université McGill, 1972. Pp. xii, vii, 144, 74. Thèses canadiennes sur microfilm, No 11855 .

Fallaize, Mgr. Pierre

Buliard, Roger. Inunuak: Mgr. Pierre Fallaize, premier missionnaire et évêque des Esquimaux du cuivre. Paris : O.P.E.R.A., 1972. Pp. 252, illus.

\section{Ferron, Marie Rose}

Une stigmatisée canadienne: Marie Rose Ferron, 1902-1936. [Saint-Javite : Éditions Magnificat, 1969.] Pp. 56, illus.

\section{Forrester, Alexander}

HARVEY, Robert. "From Pulpit to Platform: Alexander Forrester", The Nova Scotia Historical Quarterly, 2 (1972) 349-365.

\section{Fuller, Bp. Thomas Brock}

RUGGLE, Richard. "Thomas Brock Fuller - First Bishop of Niagara", Wentworth Bygones, 10 (1973) 23-36.

\section{Garnier, Abbé Louis}

PARIsÉ, Robert. "Bâtisseurs d'empires : Louis Garnier (1870-1950) : Le Curé Labelle de la Côte-Nord (1903-1950)", Saguenayensia, 14 (1972) 86-88.

\section{Grandin, Mgr. Vital-Justin}

DEMPSEY, Hugh A., ed. "A Letter from Bishop Grandin", Alberta Historical Review, 21, 1 (Winter, 1973) 8-11, illus.

\section{Grand'Maison, Jacques}

ST-Martin, Pierre. "Jacques Grand'Maison: plaidoyer pour une praxis", Aujourd'hui Québec, 8, 21 (mai, 1971) 46-150.

\section{Groulx, Chanoine Lionel-Adolphe}

GroulX, Lionel. Mes mémoires. t. 3, v. 5, Retour à l'histoire (1926-1931); v. 6, Une période laborieuse (1931-1939). Montréal : Fides.

Harris, Rev. William Richard

SCOLlARD, Robert J. "Reverend William Richard Harris, 1846-1923", Study Sessions, 41 (1974) 65-80.

\section{Henderson, John Lanceley Hodge}

WiLson, Alan. "John Lanceley Hodge Henderson", CHR, 54 (1973) 480-481.

Hine, Miss Grace L.

Hall, Alfreda. Grace to Ontario: The Story of a Baptist Missionary in Ontario, Miss Grace L. Hine. [Toronto: Baptist Resource Centre, 1972.] Pp. 74, illus. 


\section{Jones, Peter}

Easto, Mildred. "Peter Jones 1802-1856", The York Pioneer, (1973) 70-77. JONES, Peter. History of the Ojibway Indians with Especial Reference to their Conversion to Christianity. With a brief memoir of the writer and introductory notice by G. Osborn. Toronto: Canadiana House, 1973. Pp. vi, 278, illus. (Originally published London, 1861.)

\section{Kateri Tekakwitha}

BÉchaRd, Henri. "La Vénérable Kateri Tekakwitha, 1656-1680", QuébecHistoire, 1, 3-4 (juillet-déc., 1971) 34-37.

\section{Labelle, Abbé F.-X.-A.}

See above : 8A. Cornez.

Connez, Germaine. "Il y a 100 ans : La Corvée de bois du curé Labclle", Québec-Histoire, 1, 5-6 (mai-juillet, 1972) 86.

\section{Labrecque, Mgr. Michel-Thomas}

Drolet, Jean-Claude. "Le troisième évêque de Chicoutimi : Mgr. MichelThomas Labrecque (1892-1927) d'après les mémoires de Mgr. Eugène Lapointe", Saguenayensia, 13 (1971) 98-99.

Lapointe, Mgr. Eugène

Drolet, Jean-Claude. "L'œuvre sociale de Mgr. Eugène Lapointe", Saguenayensia, 13 (1971) 22-27.

\section{de Laval de Montmorency, Mgr. François}

Campeau, Lucien. "Mgr. de Laval et le Conseil souverain 1659-1684", RHAF, 27 (1973-1974) 323-359.

Vachon, André. "François de Laval, chanoine et archidiacre d'Evreux", Les cahiers des Dix, 37 (1972) 223-238.

\section{McDougall, John}

See above: 8A. Allan; McDougall.

FrIESEN, John W. "John McDougall : The Spirit of a Pioneer", Alberta Historical Review, 22, 2 (Spring, 1974) 9-17, illus.

\section{Mance, Jeanne}

D'Allaire, Micheline. "Jeanne Mance à Montréal en 1642 : Une femme d'action qui force les événements", Forces 23 (2e trimestre, 1973) 38-46.

\section{Marie de l'Incarnation}

OURY, Guy. Ce que croyait Marie de l'Incarnation et comment elle vivait sa foi. Paris: Mame, 1972. Pp. 195.

Savary, Charlotte. "300e anniversaire du décès de Marie de l'Incarnation", Québec-Histoire, 1, 5-6 (mai-juillet, 1972) 32-35.

\section{Morice, Rev. Adrien-Gabriel, O.M.I.}

HUEL, Raymond. "Adrien-Gabriel Morice, O.M.I., and the University of Saskatchewan", RUO, 43 (1973) 195-204. 


\section{O'Reilly, James}

Swainson, Donald. "James O'Reilly and Catholic Politics", Historic Kingston, 21 (1973) 11-21.

\section{Peterson, Principal William}

See above : 1 . Winkler.

\section{Racine, Mgr. Dominique}

Drolet, Jean-Claude. “Quelques enseignements de Mgr. D. Racine”, Saguenayensia, 13 (1971) 12-14.

\section{Richard, R.P. Marcel-François}

DoucET, Camille-Antonio. Une Étoile s'est levée en Acadie : Marcel-François Richard. Rogersville, N.-B. : Les Éditions du Renouveau Enr. pour les Pères Trappistes, 1973, Pp. 312.

\section{Riel, Louis}

Bowsfield, HaRTWell. Louis Riel, le patriote rebelle. Tr. de Pierre-Louis Gélinas. Montréal : Éd. du Jour, 1973. Pp. 173.

Flanagan, Thomas. "Louis 'David' Riel : prophet, priest-king; infallible pontiff", Journal of Canadian Studies/Revue d'études canadiennes, 9, 3 (August 1974 Août) 15-25.

\section{Rowat, W. A.}

Rowat, W. A. Backward glances : memoirs of a minister's son: an autobiography of a banker. 1971.

\section{Strachan, Bp. John}

PURDY, J. D. "John Strachan and the Diocesan Theological Institute at Cobourg, 1842-1852", Ontario History, 65 (1973) 113-123.

- "John Strachan's Educational Policies, 1815-1841", Ontario History, 64 (1972) 45-64.

\section{Tremblay, Abbé Alfred}

Simard, O. D. "M. l'abbé Alfred Tremblay, 1856-1921", Saguenayensia, 14 (1972) 138-141.

\section{Trivett, Rev. Samuel}

Carter, David J. "The Rev'd. Samuel Trivett", Alberta Historical Review, 21, 2 (Spring, 1973) 13-19, illus.; 21, 3 (Summer, 1973) 18-27, illus.

\section{Walsh, Mother Mary Berchmans}

Macinnis, Maria Berchmans. Keep Her Memory Bright: An Active Contemplative. Halifax : Mount Saint Vincent, [1972]. Pp. xii, 192, illus.

\section{Woodworth, Deacon Elihu}

Woodworth, Elihu. The Diary of Deacon Elihu Woodworth, 1835-1836. Transcribed by Frederick Irving Woodworth; edited by Watson Kirkconnell. [Wolfville, N.S.]: Wolfville Historical Society, 1972. Pp. 44, illus. 


\section{RELIGIOUS PRACTICE AND PASTORAL CARE}

See above : 1. Savard.

5B. Methodist-Emery; United Church-Hobbs; United ChurchPlato.

7. Légaré.

8A. Cornez.

8B. Courchesne-Bélanger; Fabre-Jetté; Ferron-Stigmatisée; Garnier-Parisé; Grandin-Dempsey; Labelle-Cornez; LapointeDrolet; Racine-Drolet.

BaUreIss, Gunter. "The Chinese Community in Calgary", Alberta Historical Review, 22, 2 (Spring, 1974) 1-8, illus.

- "The Chinese Community of Calgary", Canadian Ethnic Studies/Études ethniques du Canada, 3, 1 (June/juin, 1971) 43-56.

BÉlanger, Léonidas. "Sur le Cap Trinité : Notre Dame du Saguenay", Québec-Histoire, 1, 5-6 (mai-juillet, 1972) 95-97.

BIBEAU, Gilles. Un groupe religieux devient québécois: les bérets blancs: L'apparition et l'interprétation psycho-sociologique du phénomène. Thèse de M. Sc. Soc., Université Laval, 1973, Pp. xi, 161.

Blain, Jean. "La Moralité en Nouvelle-France : les phases de la thèse et de l'antithèse", $R H A F, 27$ (1973-1974) 408-416.

BLAND, Salem Goldworth. The New Christianity: or The Religion of the New Age. Intro. by Richard Allen. Toronto: University of Toronto Press, c1973. [First published 1920.] Pp. xxvi, 89, (The Social History of Canada.)

BRODEUR, Élisabeth Germain. Le clergé catholique du Canada: analyse sociographique/The Catholic clergy of Canada. Québec : Centre de recherche en sociologie religieuse, Faculté de théologie, Université Laval, 1973. Pp. ix, 62, ix, 62.

"Un cadre de la fonction publique. Histoire de vie", Recherches sociographiques, 14 (1973) 229-267.

Clemens, James M. "Taste Not; Touch Not; Handle Not: A Study of the Social Assumptions of the Temperance Literature and Temperance Supporters in Canada West between 1839 and 1859", Ontario History, 64 (1972) $142-160$.

DéCARIE, Malcolm Graeme. The Prohibition Movement in Ontario: 1894 1916. Ph. D. Thesis, Queen's University, 1972. Pp. 370. Canadian Theses on Microfilm, No 12706.

DE VaLK, Alphonse. "The Abortion Issue in Contemporary Canadian History : The Unfinished Debate", Study Sessions, 41 (1974) 81-99.

Donnelly, John F. "Our Church of the North”, Queens Quarterly, 80 (1973) 241-245.

Drolet, Jean-Claude. "Un mouvement da spiritualité sacerdotale au Québec au XXe siècle (1931-1965): Le Lacouturisme", Sessions d'étude, 40 (1973) 55-91. 
Dussault, Gabriel. "La religion de l'ordre... et après? Aperçus sur la morale québécoise de 1900", Relations, 377 (décembre, 1972) 330-334.

Hallowell, Gerald A. Prohibition in Ontario, 1919-1923. Toronto : Ontario Historical Society, 1972. Pp. xi, 180.

HaRveY, Julien. "L'évangélisation au Québec, 1945-1970", Communauté chrétienne, 11, 62-63 (mars-juin, 1972) 154-161.

HieberT, A. J. "Prohibition and Social Problems in British Columbia: The Example of the Okanagan", B.C. Perspectives, 2 (Oct., 1972) 36-56.

Hurtubise, $P$. "Aspects doctrinaire de la dévotion à la Sainte Famille en Nouvelle-France”, Église et Théologie, 3 (1972) 45-68.

LACRoIX, Benoît. "Dieu dans la religion populaire franco-québécoise", Communauté chrétienne, 10, 58-59 (juillet-octobre, 1971) 236-248.

Lamonde, Yvan. Louis-Adolphe Paquet. (Collection "Classiques canadiens", 45.) Montréal : Fides, 1972. Pp. 88.

LAPERRIÈRE, Guy. "L’Église et l'argent : les quêtes commandées dans le diocèse de Sherbrooke, 1893-1926", Sessions d'étude, 41 (1974) 61-85.

McCoY, John A. Orthodoxy and Change in the Roman Catholic Church. M.A. Thesis, McGill University, 1972. Pp. vi, 112. Canadian Theses on Microfilm, No 11922.

McLean, Robert Irwin. A Most Effectual Remedy: Temperance and Prohibition in Alberta, 1875-1915. M.A. Thesis, University of Calgary, 1969, Pp. 161. Canadian Theses on Microfilm, No 12786.

MaIllet, Antonine. Rabelais et les traditions populaires en Acadie. (Les Archives de Folklore, 13) Québec: Les Presses de l'Université Laval, 1971. Pp. X, 201.

Massé, Jacqueline C. "Le travail du dimanche", Sociologie et sociétés, 2 (1970) 145-161.

NORTHOVER, Wallace E. Religious Disaffection and Perceived Value Discrepancy among Roman Catholics. Ph.D. Thesis, York University, 1972. Pp. xiv, 255. Canadian Theses on Microfilm No 11438.

Nowlan, Alden. "Testimony of a Tent Preacher : fire and brimstone in the Upper St. John River valley", Maclean's Magazine, 85 (Dec., 1972) 34-35, 66.

Penny, Nancy L. Marriage Patterns in an Ethnic Community in Rural Manitoba, 1896-1970. M.A. Thesis, University of Manitoba, 1972. Pp. iii, 105.

PichetTe, Jean-Pierre. "Le curé à travers les chansons traditionnelles au Québec", Ethnologie québécoises I : 'Cahiers du Québec, Montréal : HMH, 1972, 159-184.

ROCHER, Guy. "L'incroyance comme phénomène sociologique", Communauté chrétienne, 10, 60 (novembre-décembre, 1971) 353-373.

Rousseau, L. "Une image globale des représentations de Dieu dans la théologie de Québec, au XVIII' siècle", Église et Théologie, 2 (1971) 185-195. 
Speisman, Stephen A. "Munificent Parsons and Municipal Parsimony : Voluntary vs Ptblic Poor Relief in Nineteenth Century Toronto", Ontario History, 65 (1973) 33-49.

Stryckman, Paul. Les prêtres du Québec aujourd'hui: Résultats d'une recherche sociologique. Vol. 1. Québec: Centre de recherches en sociologie religieuse, Université Laval, 1970. Pp. 260.

Stulberg, F. G. "Early Pastoral Service at York and Toronto", The York Pioneer, (1971) 43-49.

Thibault, Pierre. Savoir et pouvoir: philosophie thomiste et politique cléricale au XIX $X^{e}$ siècle. Québec: Presses de l'Université Laval, 1972. Pp. xxviii, 252.

Thomas, C. E. "The Work, in Nova Scotia, of the Society for the Propagation of the Gospel in Foreign Parts, 1784 to 1886", Collections of the Nova Scotia Historical Society, 38 (1973) 63-90.

Tremblay, Victor. "Mémoires d'une ancienne : Mademoiselle Emélie Tremblay", Saguenayensia, 13 (1971) 44-50, 108-110, 138-140; 14 (1972) 17-21.

Woodsworth, J. S. My Neighbour. With an introduction by Richard Allen. Toronto : University of Toronto Press, 1972. Pp. xx, 216.

\section{MISSIONS}

See above : 2. Journal; Laflèche; Relations.

3. Dumas.

5B. Moravian-Bruemmer; Moravian-Hiller.

8A. Allan; Copway; McDougall.

8B. Fallaize-Buliard; Hine-Hall; Jones-Easto; Jones-Jones; Kateri-Béchard; Laval-Campeau; Laval-Vachon; McDougallFriesen; Mance-D'Allaire; Marie-Oury; Marie-Savary.

9. Blain; Thomas.

BRUEMMER, Fred. "The Naskapis of Labrador", Canadian Geographical Journal, 83 (July-Dec., 1971) 94-101, illus.

CAMPEAU, Lucien. "Le dernier voyage de Champlain, 1633", TRSC/MSRC, 10 (1972) 81-101.

CARon, Ivanhoë. The 'Catholic Missions in Canada: 1721. A Profile for Genealogy and Microhistory based on a Proces-verbal by ProcureurGeneral Collet. Cottonport, Louisiana : Polyanthos, 1972. Pp. 32.

CURTIS, Edward S. In a Sacred Manner We Live : Photographs of the North American Indian. Barre, Mass. : Barre Publishers; Don Mills, Ont. : Longman Canada, 1972. Pp. 149, illus.

De Coccola, Raymond, and Paul King. Ayorama. Don Mills, Ont. : Paper Jacks, 1973, c1955. Pp. x, 316, illus.

De Grace, Eloi. "Les missionnaires et la dîme chez les Acadiens du Nouveau-Brunswick, 1790-1830", Société historique acadienne, 4, 9 (avrilmai-juin, 1973) 353-361. 
Dragon, Antonio. L'Acadie et ses 40 robes noires. Montréal : Bellarmin, 1973. Pp. 244.

Falk, Gérald Arthur. Missionary Education Work amongst the Prairie Indians, 1870-1914. M.A. Thesis, University of Western Ontario, 1973. Canadian Theses on Microfilm, No 14036.

Foster, John E. "Missionaries, Mixed-Bloods and the Fur Trade: Four Letters of the Rev. William Cockran, Red River Settlement, 1830-1833", The Western Canadian Journal of Anthropology, 3, 1 (1972) 94-125.

GARRAD, Chas. "The Attack on Ehwae in 1640", Ontario History, 65 (1973) 107-111.

Graft, Erik. L'impact de l'eau-de-vie sur les missions indiennes. Thèse de M.A., Université de Montréal, 1973, Pp. 138.

HeIDENReICH, Conrad. Huronia: A History and Geography of the Huron Indians 1600-1650. Toronto: McClelland and Stewart, 1971. Pp. 337.

HUNGRY Wolf, Adolf. Good Medicine Thoughts. Fort McLeod, Alta.: Good Medicine Books, c1972. Pp. [32], illus.

Ion, A. Hamish. Canadian Missionaries in Meiji Japan: The Japan Mission of the Methodist Church of Canada, 1873-1889. M.A. Thesis, McGill University, 1972. Pp. 220. Canadian Theses on Microfilm, No 11852.

JAENEN, Cornelius J. "French Colonial Attitudes and the Exploration of Jolliet and Marquette", Wisconsin Magazine of History, 56, 4 (Summer, 1973) $300-310$.

LaNDon, Fred. "By canoe to Lake Superior in 1838", Inland Seas, 29 (Spring, 1973) 33-36, 45-46.

Mrtchell, Estelle. "Sainte-Marie au pays des Hurons, 1639-1649 : École d'héroïsme", Québec-Histoire, 1, 3-4 (juillet-déc., 1971) 31-32.

Moon, Mary. “The O'Keefe Ranch", Okanagan Historical Society Report, 37 (1973) 101-103, illus.

PEAKe, Frank A. "Fur Traders and Missionaries: Some Reflections on the Attitudes of the Hudson's Bay Company towards Missionary Work among the Indians", The Western Canadian Journal of Anthropology, 3, 1 (1972) 72-93.

Pritchard, James S. "For the Glory of God: The Quinte Mission, 16681680", Ontario History, 65 (1973) 133-148.

Rogers, G. A. "St-Regis", Québec-Histoire, 1, 5-6 (mai-juillet, 1972) 68-71.

RoNDA, James P. "The European Indian : Jesuit Civilization Planning in New France", Church History, 41 (1972) 385-395.

Rowand, Evelyn. "The Rebellion at Lac La Biche", Alberta Historical Review, 21, 3 (Summer, 1973) 1-9.

SAGARD, Gabriel. "Le grand voyage du pays des Hurons", Écrits du Canada français, 36 (1973) 205-251.

Ste. Marie I Museum Project. General Outline. London : University of Western Ontario, [1971]. Pp. ca. 150. 
Ste. Marie I Museum Project. Storyline Manual. London : University of Western Ontario, [1971]. Pp. ca. 300.

"Ste. Marie/Visages de l'histoire vivante", Québec-Histoire, 1, 3-4 (juilletdéc., 1971) 7-11.

Salter, Robina. "The Middle Years of the Grenfell Mission : Dr. Charles S. Curtis, 1915-64", Among the Deep Sea Fishers, 70 (July, 1972) 1-3.

Simard, Robert. "La mission d'Onistagan : L'activité et la fin", Saguenayensia, 13 (1971) 69-73.

- "La mission d'Onistagan: La fondation", Saguenayensia, 13 (1971) 51-54.

Tremblay, Victor. "Des choses sur les Montagnais", Saguenayensia, 14 (1972) 169-171.

— "Deux épisodes à Tadoussac en 1629", Saguenayensia, 13 (1971) 142-144.

WARWICK, Jack. "Humanisme chrétien et bons sauvages (Gabriel Sagard, 1623-1636)", XVIIe Siècle, 97 (1972) 25-49.

\section{SPECIAL PROBLEMS}

A. The Church and the Arts

See above: 1. Catalogue.

9. Maillet; Pichette.

Bélanger, Leonidas. "Nos musiciens: Mgr. Romuald Chayer", Saguenayensia, 14 (1972) 62-66.

BRIDGE, Kay. "My Uncle Went to the Moon: An Informal Conversation with K. J. Butler", Artscanada, 184-187 (December 1973/January 1974) 154-158, illus.

Harper, J. Russell. A People's Art: Primitive, Naive, Provincial, and Folk Painting in 'Canada. Toronto: University of Toronto Press, 1974, Pp. 180 , illus.

Johnson, Nick. "Bits of Dough, Twigs of Fire", Artscanada, 184-7 (December 1973/January 1974) 61-69, illus.

LAFRAMBoISE, Yves. "L'architecture religieuse dans la vallée du Richelieu : Contribution d'Isidore Desnoyers", Docunents: Arts anciens du Québec, 2 (mai, 1971) 1-13.

Lock, William Rowland. Ontario Church Choirs and Choral Societies. D.M.A. Thesis, University of Southern California, 1972, Pp. 261. DissAbs, 33 (1972-73) 5767-A.

McDougall, Charlotte. "Saint-Jean-Port-Joli", Canadian Geographical Jounnal 82 (Jan.-June, 1971) 108-112, illus.

MacNaIr, Peter L. "Kwakiute Winter Dances : A Reenactment", Artscanada, 184-7 (December 1973/January 1974) 94-114.

— "Potlatch at Alert Bay", Artscanada, 184-7 (December 1973/January 1974) 115-118. 
MARWICK, William Edward. The Sacred Choral Music of Healey Willan. Ph.D. Thesis, Michigan State University, 1970. Pp. 278.

Maugey, Axel. "La présence de Dieu dans la poésie québécoise", Communauté chrétienne, 10, 58-59 (juillet-octobre, 1971) 248-262.

MUSÉE DU QUÉBEC. Trésors des communautés religieuses de la ville de Québec. Québec : Éd. officiel du Québec, 1973. Pp. 199.

NOPPEN, Luc. "Raphaël Giroux 1815-1869 sculpteur et architecte", $A u$ fil des événements, 8, 22 (1er mars 1973) 9.

Oвomsawin, Alanis. "Sounds and Voices from our People", Artscanada, 184-7 (December 1973/January 1974) 81-89, illus.

OstiguY, Jean-René. Étude sur des dessins préparatoires à la décoration du baptistère de l'église Notre-Dame-de-Montréal. Bulletin 15, Ottawa : Galerie Nationale du Canada, 1971.

OTTo, Eberhard. "Sacred Structures, Sea and Forest : Queen Charlotte Islands", Artscanada, 184-187 (December 1973/January 1974) 119-124, illus.

PORTER, John R. et Léopold Désy. Calvaires et croix de chemins du Québec. Montréal : HMH, 1973. Pp. 256, illus.

Proulx, L. The Mosaic Pavements of Saint Ann's Basilica. [Sainte-Anne-deBeaupré : Saint Ann's Basilica, 1973.] Pp. 24, illus.

- Le pavement de la basilique Sainte Anne. [Sainte-Anne-de-Beaupré : Basilique Sainte-Anne, 1973.] Pp. 24, illus.

RICHER, Laurette B. "Les chapelles d'Oka", Québec-Histoire, 1, 5-6 (maijuillet, 1972) 46-48.

Rothenberg, Jerome, and Dennis Tedlock. "The Shaman as Proto-Poet : An Anthology of Shamanic Texts and Commentary",Artscanada, 184-187 (December 1973/January 1974) 172-181, illus.

Simard, Jean. "Témoins d'un passé de foi", Perspectives-Le Soleil, 14, 25 (17 juin, 1972) 20-22, illus.

Vastokas, Joan, and Romas Vastokas. Sacred Art of the Algonkians. Peterborough, Ontario : Mansard Press, 1973. Pp. 178.

VASTOKAS, Joan M. "The Shamanic Tree of Life", Artscanada, 184-187 (December 1973/January 1974) 125-149, illus.

WebBer, Alika Podolinsky. "The Healing Vision: Naskapi Natutshikans", Artscanada, 184-187 (December 1973/January 1974) 150-153, illus.

Woodcock, George. "Callaghan's Toronto: the Persona of a City", Journal of Canadian Studies/Revue d'études canadiennes, 7, 3 (August 1972 août) 21-24.

\section{B. The Church and Education}

See above: 6A. Chicoutimi-Bélanger; Chicoutimi-Drolet (3); ChicoutimiGerbe; Chicoutimi-Laiiberté; Chicoutimi-Paré (2); Chicoutimi-Pedneault; Chicoutimi-Simard (2); Chicoutimi-Tremblay (4); Kingston-Flynn. 
7. Audet; Begnal; Carrière (2); DeWolf; Frères; McGill; Jeanne; Leefe; Neatby; O'Farrell; Pariseau; Penton; Trudel.

8A. Houde.

8B. Casault-Sylvain; Drolet-Boissonnault; Groulx-Groulx; Henderson-Wilson; Morice-Huel; Strachan-Purdy (2).

10. Falk.

Charbonneau, Jean Claude. The Lay School Movement in Quebec since 1840. M.A. Thesis, McGill University, 1972. Pp. iv, 166. Canadian Theses on Microfilm, No 11778.

Drolet, Jean-Claude. "Un parlement-modèle au Séminaire de Chicoutimi en 1912-1913”, Saguenayensia, 14 (1972) 79-83, 93-96.

Ecumenical Institute of Canada, Study Commission. Religion in our Schools: An Ecumenical Reaction to the Keiller Mackay Report. Toronto : Ecumenical Study Commission, [1972]. Pp. 28.

Fingard, Judith. "Attitudes toward the Education of the Poor in Colonial Halifax", Acadiensis, 2, 2 (Spring, 1973) 15-42.

GidNEY, R. D. "Centralization and Education: The Origins of an Ontario Tradition", Journal of Canadian Studies/Revue d'études canadiennes, 7, 4 (November 1972 novembre) 33-48.

Hatfield, Michael Frederick. La Guerre scolaire: the Conflict over the New Brunswick Common Schools Act, 1871-1876. M.A. Thesis, Queen's University, 1972. Pp. xiv, 185. Canadian Theses on Microfilm, № 11706.

Huel, Raymond J. "The Teaching of French in Saskatchewan Public Schools : A curious infraction of the provisions of the School Act in Ethier, S.D. No 1834, 1921-23". Saskatchewan History, 24 (1971) 13-24.

LAJeUnesse, Marcel. L'éducation au Québec (19e-20e siècles). Montréal : Les éditions du Boréal Express, 1971. Pp. 145.

LAMONDE, Yvan. "Classes sociales, classes scolaires: une polémique sur l'éducation en 1819-1820", Sessions d'étude, 41 (1974) 43-59.

LAWR, Douglas, and Robert GIDNeY, eds. The Canadian Education Tradition : a documentary history. Toronto: Van Nostrand, Reinhold, 1972.

MCCreath, P. L. "Charies Tupper and the Politics of Education in Nova Scotia", The Nova Scotia Historical Quarterly, 1 (1971) 203-225.

Majerus, Yvette Viviane. L'éducation dans le diocèse de Montréal d'après la correspondance de ses deux premiers évêques, Mgr. J.J. Lartigue et Mgr. J. Bourget, de 1820 à 1867. Thèse de Ph.D. Université McGill, 1971. Pp. xiv, 228. Thèses canadiennes sur microfilm, No 11417.

Mathews, Barbara Lee Cannon. The Growth of Disagreement among Teachers over the Dual School System in the Province of Quebec. Ph.D. Thesis, The University of Michigan, 1973. Pp. 323. DissAbs, 34 (197374) 4814-A.

Mitchell, Malcolm, Gailand MacQueen, Marina Bieler. Three Approaches to Religious Education. Toronto: Ontario Institute for Studies in Education, c1972. Pp. vi, 30. 
PoIRIER, Pascal. "La loi scolaire de 1871 au N.-B. et ses répercussions", La Société Historique Acadienne, 34, 4 (janvier-mars, 1972) 141-152. (Written in 1916.)

PuXIEY, H. L. Editor. "Religious Education in a Pluralistic Society", Addresses and Papers of the National Conference, Ecumenical Study Commission, Toronto : Canada, May 29-June 1, 1973. Religious Education, 68, 4-5 (July-August, 1973).

Steele, Catherine. "Frances Ridley Havergal and Havergal College", The York Pioneer, (1973) 36-45.

Thомas, Theodore Elia. The Protestant Churches and the Religious Issue in Ontario Public Schools: A Study in Church and State. Ph. D. Thesis, Columbia University, 1972. Pp. 355. DissAbs, 33 (1972-73) 6444-A.

\section{The Church and Immigration and Settlement}

See above : 3. Burnet; Norris; Palmer.

8A. Allan; McDougall.

8B. McDougall-Friesen.

9. Baureiss (2)

10. Campeau; Jaenen; Landon; Moon; Ronda; Rowand; Sagard.

CURrie, L. D. "The Emigrants of St. Ann's", Collections of the Nova Scotia Historical Society, 38 (1973) 113-126.

Larouche, Fernand. "L'immigrant dans une ville minière. Une étude de l'interaction', Recherches sociographiques, 14 (1973) 203-228.

Mannion, John J. Irish Settlements in Eastern 'Canada: A Study of Cultural Transfer and Adaptation. Pp. 220, illus.

MARUNCHAR, Mychailo H. Study do Istoriyi Ukrainciy Kanady/Studies in the History of Ukrainians in Canada, Vol. 4: Sketches, Articles, Essays to the Pioneers' Era. Winnipeg: Ukrainiar Free Academy of Sciences, 1972.

SUNTER, Ronald. "The Scottish Background to the Immigration of Bishop Alexander Macdonnell and the Glengary Highlanders", Study Sessions, 40 (1973) 11-20.

\section{The Church and Politics}

See above : 2. Dessaules.

5B. Quaker-Fell.

7. Guitard.

8B. Forrester-Harvey; Laval-Campeau; O'Reilly-Swainson; RielBowsfield; Riel-Flanagan.

9. de Valk, Thibault.

10. Rowand.

11B. Hatfield; McCreath; Poirier; Thomas.

BAKER, W. M. "Turning the Spit: Timothy Anglin and the Roasting of D'Arcy McGee", HPCH, (1974) 135-155.

BÉlANGER, André-Jacques. L'apologétisme des idéologies québécoises et le grand tournant de 1934-1936. Thèse de D.Sc.Soc., Université Laval, 1973. Pp. v, 757. 
BERNARD, Jean-Paul, comp. Les idéologies québécoises au $19 e$ siècle. Montréal : Éditions du Roréal Express, 1973. Pp. 149.

- Les Rouges: Libéralisme, nationalisme et anticléricalisme au milieu du $X I X^{e}$ siècle. Montréal : Les Presses de L'Université du Québec, 1971. Pp. xx, 395.

Cameron, James M. "Fenian Times in Nova Scotia", Collections of the Nova Scotia Historical Society, 37 (1970) 103-152.

СоноE, Margaret. "Shannon's Cannon”, Historic Kingston, 22 (1974) 60-63.

Crunican, Paul. Priests and Politicians: Manitoba Schools and the Election of 1896. Toronto : University of Toronto Press, 1974. Pp. 370.

Denault, J.-Raymond, et Maurice Marquis. "Centenaire de 'Nos Croisés' : Les Zouaves Pontificaux canadiens”, Québec-Histoire, 1, 3-4 (juillet-déc., i 971$)$ 64-67.

De ValK, A. Morality and Law in Canadian Politics: The Abortion Controversy. Montreal: Palm Publishers, 1974. Pp. 184.

- The Unfinished Debate: The Abortion Issue in Contemporary Canadian History. [Toronto : Life Cycle Books, 1974.] Pp. 15.

Drolet, Jean-Claude. "Un affrontement libéral-ultramontain au diocèse de Chicoutimi en 1888", Saguenayensia, 13 (1971) 74-76.

Durocher, René. "Henri Bourassa, les évêques et la guerre de 1914-1918", Communications historiques, (1971) 248-275.

Gache, Paul. "La bataille de Loigny", Québec-Histoire, 1, 3-4 (juillet-déc., 1971) 59-63.

JoNES, Richard R. "L'idéologie de l'Action catholique", RHAF 27 (1973-74) 63-76.

Kulisek, Larry Lee. D'Alton McCarthy and the True Nationalization of Canada. Ph. D. Thesis, Wayne State University, 1973. Pp. 492. Diss Abs, 34 (1973-74) 3307-A.

MacDermaid, Anne. "The Visit of the Prince of Wales to Kingston in 1860", Historic Kington, 21 (1973) 50-61.

Middlebro, Tom. "The French Fact, Catholicism and the N.D.P." RUO, 43 (1973) 566-572.

MilleR, J. R. "D'Alton McCarthy, Equal Rights, and the Origins of the Manitoba School Question", 'CHR, 54 (1973) 369-392.

- "Equal Rights for All': The E.R.A. and the Ontario Election of 1890", Ontario History, 65 (1973) 211-230.

- "Honoré Mercier, la minorité protestante du Québec et la loi relative au règlement de la question des biens des Jésuites", $R H A F, 27$ (19731974) 483-507.

- The Impact of the Jesuits' Estates Act on Canadian Politics, 18881891. Ph. D. Thesis, University of Toronto, 1972. DissAbs, 34 (197374) 5069-A.

- "The Jesuits' Estates Act crisis: 'An incident in a conspiracy of several years' standing'", Journal of Canadian Studies/Revue d'études canadiennes, 9,3 (August 1974 août) 36-50. 
- "This Saving Remnant': Macdonald and the Catholic Vote in the 1891 Election", Study Sessions, 41 (1974) 33-52.

Olssen, Andrée Lévesque. The Canadian Left in Quebec during the Great Depression: The Communist Party of Canada and the Co-Operative Commonwealth Federation in Quebec, 1929-1939. Ph.D. Thesis, Duke University, 1973. Pp. 259. DissAbs, 34 (1973-74) 708-A.

Tremblay, Jean-Paul. Napoléon Aubin. Textes choisis et présentés. (Collection "Classiques canadiens", 43) Montréal: Fides, 1972. Pp. 95.

Wallot, Jean-Pierre. "The Lower-Canadian Clergy and the Reign of Terror (1810)", Study Sessions, 40 (1973) 53-60.

\section{E. The Church and Society}

See above : 1. Bissonnette; Leblanc.

3. Paquet; Russell.

7. Agnew; D'Allaire; Desjardins (2); Drolet (3); Gibson; Hôtel-Dieu; La Palm; Maryan.

8A. Cornez.

8B. Garnier-Parisé; Labelle-Cornez; Lapointe-Drolet; ManceD'Allaire; Richard-Doucet.

9. Baureiss (2); Bland; Brodeur; Clemens; Decarie; de Valk; Hallowell; Hiebert; McLean; Massé; Penny; Rocher; Speisman; Stryckman; Woodsworth.

10. Foster; Peake; Rowand; Salter.

11A. Woodcock.

11B. Fingard; Lamonde.

11D. de Valk (1) (2); MacDermaid.

ANDERson, Alan Betts. Assimilation in the Bloc Settlements of North-Central Saskatchewan: A Comparative Study of Identity Changes among Seven Ethno-Religious Groups in a Canadian Prairie Region. Ph.D. Thesis, University of Saskatchewan, 1972. Pp. 415. DissAbs, 33 (1972-73) 3034-A.

ARÈs, Richard. "La sécularisation de la société québécoise", Relations, 353 (octobre, 1970) 274-277.

Avery, Donald Howard. Canadian Immigration Policy and the Alien Question, 1896-1919: The Anglo-Canadian Perspective. Ph.D. Thesis, University of Western Ontario, 1973. Diss Abs, 34 (1973-74) 1806-A.

Baillargeon-Pelletier, Hélène. "Les Chartrand: trente ans du Québec", Maintenant, 109 (octobre, 1971) 260-285.

Cross, Michael S. "The Shiners' War : Social Violence in the Ottawa Valley in the 1830's", CHR, 54 (1973) 1-26.

Delude-Clift, Camille et Édouard Champoux. "Le Conflit des générations", Recherches sociographiques, 14 (1973) 157-201.

Desrosiers, Richard et Denis Héroux. Le travailleur québécois et le syndicalisme. 2e édn. rev. et corrigée, mise à jour. Montréal: Presses de l'Université du Québec. 1973. Pp. 156. 
HARVEY, Fernand. Aspects historiques du mouvement ouvrier au Québec. Montréal : Éd. du Boréal Express, 1973. Pp. 227.

HoRn, Michael. "The League for Social Reconstruction and the Development of a Canadian Socialism, 1932-1936", Journal of Canadian Studies/Revue d'études canadiennes, 7, 4 (November 1972 novembre) 3-17.

Maxwell, Thomas R. "La population d'origine française de l'agglomération Métropolitaine de Toronto", Recherches sociographiques, 12 (1971) 319-344.

Olthuis, James H., and Gerald VANDEZANDE. Bunglers and Visionaries: Christian Labour at the Crossroads: Essays. Toronto: Wedge Pub. Foundation, c1972. Pp. iii, 38.

PALMER, Howard. "Nativism in Alberta, 1925-1930", HPCH, (1974) 183-212.

Rothwell, David R. "United Church Pacifism-October, 1939", The Bulletin, 22 (1973) 36-55.

Sellar; Robert. The Tragedy of Quebec: The expulsion of its Protestant Farmers, 1916. Introduction by Robert Hill. (Social History of Canada Series, No 17.) Toronto: University of Toronto Press, 1974. Pp. 374.

Sylvain, Philippe. "Les Chevaliers du travail et le cardinal Elzéar-Alexandre Taschereau", TRSC/MSRC, 11 (1973) 31-42. 